\title{
The well-rounded linear function
}

\author{
Roger B. Eggleton \\ Mathematics Department, Illinois State University \\ Normal, IL 61790-4520, USA \\ roger@math.ilstu.edu \\ To Aviezri Fraenkel, friend and mentor of many years.
}

\begin{abstract}
The generic linear function $a x+b$ of a real variable, with $a, b, x \in \mathbb{R}$, is usually evaluated as a scale function (product) followed by a translation (sum). Our main result shows that when such a function is variously combined with rounding functions (floor and ceiling), exactly 67 inequivalent rounded generic linear functions result, of which 38 are integer-valued and 29 are not. Several related results are also established, with elucidation of the relevant equivalence class structures.
\end{abstract}

Submitted: June 27, 2000; Accepted: August 15, 2000.

Mathematical Reviews Subject Classification (2000): 05A15, 11A99, 26 A09.

\section{Introduction}

The floor function $\lfloor\cdot\rfloor$ and ceiling function $\lceil\cdot\rceil$ are familiar integer-valued functions of a real variable which may be collectively referred to as rounding functions. They are defined for all $x \in \mathbb{R}$ by

$$
\begin{array}{ll}
\lfloor x\rfloor=n & \text { precisely when } n \leq x<n+1 \text { and } n \in \mathbb{Z}, \\
\lceil x\rceil=n & \text { precisely when } n-1<x \leq n \text { and } n \in \mathbb{Z} .
\end{array}
$$

The half-bracket notation and the names "floor function" and "ceiling function" were introduced by Iverson [8] in a book on programming published in 1962. The floor function is identical with the integer part function [.], a terminology and notation still widely encountered in number theory. I am not sure when or by whom this notation was introduced. Dickson, for example, used it when describing Legendre's result for the power of a prime in a factorial (see [2], page 263), but Legendre (see [11], page 10) actually used the notation $E(\cdot)$, taking the initial $E$ from the French "entier". The ceiling function is 
identical with the so-called post office function, which rounds intermediate weights up to the next scale point for postal charges. Many notations have denoted it, but none was widely adopted prior to Iverson's half bracket notation. A charming discourse on the rounding functions is given in Chapter 3 of [5].

In contexts such as number theory, combinatorial game theory, and other branches of discrete mathematics, it is not uncommon to find need or convenience motivating the use of a combination of one or more rounding functions with some other type of function. For example, rounding of rational functions is used to compute the power of a prime in a factorial, and using inclusion-exclusion to count the number of primes below a specified bound (both applications that go back at least to Legendre). Rounding of exponential functions gives a convenient expression for the quadratic residue character of the prime 2 (see [7], page 75), and for the $n$th Fibonacci number (see [5], page 286). Multiple roundings of polynomial functions have been studied by Fraenkel [4], and by Håland [6] . Indeed, the title of Fraenkel's paper is in itself a brief guide to mathematical areas where rounded functions have played a significant role in recent developments.

At first glance it might appear that combining rounding functions with linear functions would have comparatively little potential interest. However, there is a considerable literature on Beatty sequences, which arise from just one rounding of a linear function: for fixed positive reals $a$ and $b$, the Beatty sequence $B(a, b)$ is the sequence of values assumed by the function $\lfloor a x+b\rfloor$ as $x$ runs through the positive integers (see [3], [4]). Beatty sequences originated with a 1927 problem [1] in the American Mathematical Monthly. Related problems keep appearing (see [9]). More than one rounding can be combined with a linear function, to produce functions like $\lceil a\lfloor x\rfloor+b\rceil$. This motivates us to ask a very basic question: How many distinct rounded linear functions can arise from a given linear function? The answer turns out to be rather larger than might be initially expected, and correspondingly the investigation is richer than expected.

To make our objective precise, we need to make a distinction which is rather unfamiliar, because it is not usually of consequence. Our need is to distinguish between the form of a function and the functions which are instances of that form. Specifically, we shall regard any function $x \mapsto a x+b$, in which $a, b \in \mathbb{R}$ are fixed and $x \in \mathbb{R}$ runs over all the reals, as an instance of the function $(a, b, x) \mapsto a x+b$, in which each of $a, b, x \in \mathbb{R}$ runs over all the reals. In the latter $a$ and $b$ are conventionally parameters, while in the former they are particular though unspecified real numbers. We define the generic linear function, or more explicitly, the generic real-valued linear function of a single real variable and two real parameters, to be the function $L: \mathbb{R}^{3} \rightarrow \mathbb{R}$ specified by $L(a, b, x)=a x+b$, and we define a linear function, or more explicitly, a real-valued linear function of a single real variable, to be a function $f_{a, b}: \mathbb{R} \rightarrow \mathbb{R}$ specified by $f_{a, b}(x)=a x+b$, for fixed $a, b \in \mathbb{R}$. Writing $f_{a, b}=L(a, b, \cdot)$ indicates this relationship. For convenience we admit the possibility $a=0$, and thus regard any constant function $f_{0, b}(x)=b$ as a (degenerate) linear function.

In the next section we shall define rounded generic linear functions and rounded linear functions, and specify when two such functions are equivalent. Our main objectives will be to count and describe the equivalence classes of rounded generic linear functions. 


\section{Parenthesis-free notation}

Using a parenthesis-free explicit notation gives us a very precise way to define and describe the rounded functions we wish to study. We shall mostly use the postfix or reverse Polish notation (e.g. see Section 2.3.2 of [10]), returning to more traditional notation to express the main results. Thus the specification $L(a, b, x)=a x+b$ for the generic linear function becomes $L(a, b, x)=a x * b+$. We wish to incorporate various rounding functions into the structure of this function. Instead of pairs of half-brackets, it is helpful in this context to have single symbols for the rounding functions. We shall use $D$ for rounding down (the floor function) and $U$ for rounding up (the ceiling function), together with $I$ for the identity function.

Before we define rounded versions of the generic linear function, first note that the composition of two rounding functions is always a rounding function. Specifically, in reverse Polish notation the composition of two functions $R, R^{\prime}: \mathbb{R} \rightarrow \mathbb{R}$, such that $R, R^{\prime} \in$ $\{I, D, U\}$, always satisfies $x R R^{\prime}=x R^{\prime \prime}$ with $R^{\prime \prime}=R^{\prime}$ if $R=I$ and $R^{\prime \prime}=R$ if $R \neq I$. Hence we gain sufficient generality by defining any rounded generic linear function, or more explicitly, any rounded generic real-valued linear function of a single real variable and two real parameters, to be any function $L: \mathbb{R}^{3} \rightarrow \mathbb{R}$ specified by

$$
L(a, b, x)=a R_{0} x R_{1} * R_{2} b R_{3}+R_{4}
$$

where $R_{i} \in\{I, D, U\}, 0 \leq i \leq 4$. It is natural to denote $L$ by its operator string (with the above convention regarding placement of the arguments $a, b$ and $x$ ), thus

$$
L=R_{0} R_{1} * R_{2} R_{3}+R_{4} .
$$

For example, the specification $L(a, b, x)=\lceil a\lfloor x\rfloor+b\rceil$ becomes $L(a, b, x)=a I x D * I b I+U$, with the function itself denoted by $L=I D * I I+U$. It is convenient to refer to such functions as roundings of the generic linear function.

Two rounded generic linear functions are formally distinct if their operator strings are different, so there are $3^{5}=243$ such functions. Two such functions $L_{0}, L_{1}: \mathbb{R}^{3} \rightarrow \mathbb{R}$ are equivalent if $L_{0}(a, b, x)=L_{1}(a, b, x)$ for every $(a, b, x) \in \mathbb{R}^{3}$, and in this case we write $L_{0} \sim L_{1}$. Thus our main question becomes: How many equivalence classes comprise the 243 formally distinct roundings of the generic linear function $L(a, b, x)=a x * b+$, and what is the structure of those equivalence classes?

If we evaluate a rounded generic linear function $L=R_{0} R_{1} * R_{2} R_{3}+R_{4}$ at any specific pair of parameters $(a, b) \in \mathbb{R}^{2}$, the result is a rounded linear function $f_{a, b}=L(a, b, \cdot)$, so $f_{a, b}: \mathbb{R} \rightarrow \mathbb{R}$ is specified by

$$
f_{a, b}(x)=a R_{0} x R_{1} * R_{2} b R_{3}+R_{4} .
$$

Two such functions $f_{a, b}=L_{0}(a, b, \cdot)$ and $g_{a, b}=L_{1}(a, b, \cdot)$ are equivalent if $f_{a, b}(x)=g_{a, b}(x)$ for every $x \in \mathbb{R}$, and then we write $f_{a, b} \sim g_{a, b}$. If $L_{0} \sim L_{1}$ then $f_{a, b} \sim g_{a, b}$ must hold, but the converse need not be true. For example, let $L_{0}=D I * I I+D$ and $L_{1}=U I * I I+D$. Then $L_{0}(1.5,1,1.5)=2$ and $L_{1}(1.5,1,1.5)=4$, so $L_{0} \nsim L_{1}$. But for $f_{a, b}=L_{0}(a, b, \cdot)$ and 
$g_{a, b}=L_{1}(a, b, \cdot)$ we have $f_{1, b}(x)=\lfloor x+b\rfloor=g_{1, b}(x)$ for all $x \in \mathbb{R}$, so $f_{1, b} \sim g_{1, b}$ for every $b \in \mathbb{R}$. Thus, our main question has a corresponding "local" version: For fixed $(a, b) \in \mathbb{R}^{2}$, how many equivalence classes comprise the 243 formally distinct roundings of the linear function $f_{a, b}(x)=a x * b+$, and what is the structure of those equivalence classes?

\section{Rounded scale functions}

Let us begin with scale functions. The generic scale function, or more explicitly, the generic real-valued scale function of a single real variable and one real parameter, is the function $S: \mathbb{R}^{2} \rightarrow \mathbb{R}$ specified by $S(a, x)=a x$, or $S(a, x)=a x *$ in reverse Polish notation. Its roundings are all the functions specified by

$$
S(a, x)=a R_{0} x R_{1} * R_{2}
$$

where $R_{i} \in\{I, D, U\}, 0 \leq i \leq 2$. Reverting to operator strings, we have $S=R_{0} R_{1} * R_{2}$. Evidently there are $3^{3}=27$ formally distinct roundings of the generic scale function. Let us consider their equivalence classes.

The function $R_{0} R_{1} * R_{2}$ is always integer-valued if $R_{2} \in\{D, U\}$, and also if $R_{2}=I$ and $R_{0}, R_{1} \in\{D, U\}$. Thus, there are $3^{2} \times 2+2^{2} \times 1=22$ formally distinct rounded generic scale functions which are integer-valued. This leaves $27-22=5$ which are formally distinct and noninteger-valued. When $(a, x)=(5.26,7.74)$, for example, the 27 formally distinct functions take on 12 different integer values and 5 different noninteger values, all within the interval [35..48], which contains just 14 integers. Hence the 5 nonintegervalued functions are all inequivalent, and the integer-valued functions belong to at least 12 equivalence classes.

Note that if $A, B \in\{D, U\}$ then

$$
A B * D \sim A B * U \sim A B * I,
$$

so the $2^{2} \times 3=12$ integer-valued functions just described belong in equivalence classes of size at least 3 . Hence the 22 formally distinct integer-valued rounded generic scale functions comprise at most 14 equivalence classes. When $(a, x)=(10.32,20.82)$, for example, these functions do take on 14 different integer values within the interval [200..231]. This confirms that there are no further equivalences, and the 12 functions described above do belong to 4 equivalence classes of size 3 : it is natural to take $A B * I$ as the representative of its equivalence class. Hence, reverting to traditional notation, we have

Theorem 1. The generic scale function $S(a, x)=$ ax has 19 inequivalent roundings. They form 14 equivalence classes of integer-valued functions (10 singletons and 4 classes of size 3), and 5 equivalence classes of noninteger-valued functions (all singletons). 
TABLE 1: Roundings of $S(a, x)=a x$

Total: 19 functions

Integer-valued functions

Total: 14 functions

10 singletons:

$\lfloor a x\rfloor,\lceil a x\rceil$,

$\lfloor a\lfloor x\rfloor\rfloor,\lceil a\lfloor x\rfloor\rceil,\lfloor a\lceil x\rceil\rfloor,\lceil a\lceil x\rceil\rceil$,

$\lfloor\lfloor a\rfloor x\rfloor,\lceil\lfloor a\rfloor x\rceil,\lfloor\lceil a\rceil x\rfloor,\lceil\lceil a\rceil x\rceil$.

4 classes of size 3 :

$\lfloor a\rfloor\lfloor x\rfloor,\lfloor a\rfloor\lceil x\rceil,\lceil a\rceil\lfloor x\rfloor,\lceil a\rceil\lceil x\rceil$.
Noninteger-valued functions

Total: 5 functions

5 singletons:

ax,

$a\lfloor x\rfloor, a\lceil x\rceil,\lfloor a\rfloor x,\lceil a\rceil x$.

A representative of each of the 19 equivalence classes is given in traditional notation in Table 1. The generic scale function with integer parameter, $S: \mathbb{Z} \times \mathbb{R} \rightarrow \mathbb{R}$ defined by $S(n, x)=n x *$, is also of interest. Because $D \sim U \sim I$ when restricted to $\mathbb{Z}$, the restriction of the parameter to $\mathbb{Z}$ results in clusters of three formally distinct functions becoming equivalent. From Table 1 we immediately deduce

Corollary 1.1. The generic scale function $S(n, x)=n x$, with integer parameter $n$, has 5 inequivalent roundings. They form 4 equivalence classes of integer-valued functions (2 classes of size 3 and 2 of size 9) and one size 3 equivalence class of noninteger-valued functions. The classes have representatives $\lfloor n x\rfloor,\lceil n x\rceil, n\lfloor x\rfloor, n\lceil x\rceil$ and $n x$, respectively.

There are situations when it is appropriate to distinguish between roundings of a function in which the last ("outermost") operator is the identity function and those in which it is one of the two rounding functions. Let us call the former mid roundings and the latter final roundings. In particular, there are $3^{2}=9$ formally distinct mid rounded generic scale functions $R_{0} R_{1} * I \sim R_{0} R_{1} *$, including the generic scale function itself (when $R_{0}=R_{1}=I$ ), and there are $3^{2} \times 2=18$ formally distinct final rounded generic scale functions $R_{0} R_{1} * A$, where $A \in\{D, U\}$. The above results then yield

Corollary 1.2. The generic scale function $S(a, x)=$ ax has 9 inequivalent mid roundings (all with singleton equivalence classes) and 14 inequivalent final roundings (comprising 10 singletons and 4 equivalence classes of size 2). Exactly 4 mid roundings are integer-valued, and each is equivalent to two of the final roundings.

Our calculations raise various open questions. For example, is there any $(a, x) \in \mathbb{R}^{2}$ for which the 14 integer-valued roundings of the generic scale function $S(a, x)=a x$ take on more than 12 values within 14 consecutive integers? What is the length of a smallest interval in which the 14 integer-valued roundings take on 14 distinct values? Is it true that in such an interval all 19 inequivalent roundings take on 19 distinct values? 
The rounded scale functions are "localized" roundings of the generic scale function. They have the form $f_{a}=S(a, \cdot)$ for some fixed value of the parameter $a \in \mathbb{R}$, where $S=R_{0} R_{1} * R_{2}$ is a rounding of the generic scale function. The equivalence classes of all such functions with the same value of $a \in \mathbb{R}$ can be deduced from Table 1 , but attention to detail is needed. Here is a brief discussion, omitting most of the details.

First note that for any fixed real $r>0$ the spectrum of equivalence class sizes when $a=-r$ is the same as when $a=r$, but the membership of the classes is different, because $\lceil-x\rceil=-\lfloor x\rfloor$ and $\lfloor-x\rfloor=-\lceil x\rceil$ hold for all $x \in \mathbb{R}$. Since all rounded scale functions with $a=0$ are equivalent to the zero function, it suffices to restrict attention to those with $a=r>0$. When $r \in \mathbb{Z}^{+}$, if $r \geq 2$ there are 5 equivalence classes as specified by Corollary 1.1 , and if $r=1$ there are 2 equivalence classes of size 12, with representatives $\lfloor x\rfloor$ and $\lceil x\rceil$, and one class of size 3, with representative $x$ (so $D, U$ and $I$ are the inequivalent scale functions in this case).

Now suppose $r \in \mathbb{R} \backslash \mathbb{Z}, r>0$. A theorem of McEliece (see [5], page 71) asserts that any continuous strictly increasing function $f: \mathbb{R} \rightarrow \mathbb{R}$ with $f^{-1}(\mathbb{Z}) \subseteq \mathbb{Z}$ has the dual properties $\lfloor f(x)\rfloor=\lfloor f(\lfloor x\rfloor)\rfloor$ and $\lceil f(x)\rceil=\lceil f(\lceil x\rceil)\rceil$. Hence $\lfloor r x\rfloor=\lfloor r\lfloor x\rfloor\rfloor$ and its dual hold for all $x \in \mathbb{R}$ when $r=1 / n$ is a unitary fraction. If $r$ is not a unitary fraction these functions are not equivalent, since there exist $k, m \in \mathbb{Z}^{+}$such that $m-1<k r<m<(k+1) r$, so $\lfloor r x\rfloor=m$ and $\lfloor r\lfloor x\rfloor\rfloor=m-1$ when $x=m / r$, and similarly for the dual. Thus, when $0<r<1$, five equivalence classes coalesce into one class of size 9 in which all members are equivalent to the zero function, two singletons coalesce with two classes of size 3 to form two classes of size 4 with representatives $\lfloor x\rfloor$ and $\lceil x\rceil$, and the remaining classes are all singletons if $r$ is not a unitary fraction, but when $r$ is such a fraction two pairs of singletons coalesce to form two classes of size 2 . When $1<r<2$, two singletons coalesce with two classes of size 3 to form two classes of size 4 with representatives $\lfloor x\rfloor$ and $\lceil x\rceil$; the remaining classes are 13 singletons and two classes of size 3. Finally, when $r>2$ there is no coalescence of equivalence classes. To summarize, we have

Corollary 1.3. For fixed $a \in \mathbb{R}$, the scale function $f_{a}(x)=$ ax has 27 formally distinct roundings, which form $N(a)$ equivalence classes: $N(a)=1$ if $a=0 ; N(a)=3$ if $|a|=1$; $N(a)=5$ if $|a| \geq 2, a \in \mathbb{Z} ; N(a)=11$ if $0<|a|<1$ and $a$ is a unitary fraction; $N(a)=13$ if $0<|a|<1$ and $a$ is not a unitary fraction; $N(a)=17$ if $1<|a|<2$; and $N(a)=19$ if $|a|>2$ and $a \in \mathbb{R} \backslash \mathbb{Z}$.

In the remainder of this paper no further explicit discussion will be devoted to equivalence classes of "localized" functions. These are of secondary interest compared with the generic functions, and the preceding discussion indicates how it is possible to determine "local" equivalence classes from "generic" ones.

\section{Rounded translations}

Next let us consider translations. The development parallels that for scale functions in the previous section, but the equivalence class structure turns out to be coarser. The generic 
translation, or more explicitly, the generic real-valued translation function of a single real variable and one real parameter, is the function $T: \mathbb{R}^{2} \rightarrow \mathbb{R}$ specified by $T(b, x)=b+x$, or $T(b, x)=b x+$ in reverse Polish notation. Its roundings are all functions of the form

$$
T(b, x)=b R_{0} x R_{1}+R_{2}
$$

where $R_{i} \in\{I, D, U\}, 0 \leq i \leq 2$, and we write $T=R_{0} R_{1}+R_{2}$. Again there are $3^{3}=27$ formally distinct roundings of the generic translation.

As with rounded generic scale functions, there are 22 formally distinct rounded generic translations which are integer-valued and 5 which are not. The former are the 18 final roundings $R_{0} R_{1}+R_{2}$ with $R_{2} \in\{D, U\}$, together with the 4 mid roundings in which $R_{2}=I$ and $R_{0}, R_{1} \in\{D, U\}$. The remaining $5 \mathrm{mid}$ roundings are the formally distinct noninteger-valued functions. When $(b, x)=(1.2,3.1)$, for example, the 27 formally distinct roundings of the generic translation take on 3 integer values and 5 noninteger values within the interval [4..6]. Hence the 5 noninteger-valued functions are all inequivalent, so they belong in 5 equivalence classes which are singletons. Furthermore, the integer-valued functions must belong to at least 3 equivalence classes.

If $A, B \in\{D, U\}$ a little reflection confirms the equivalences

$$
A B+D \sim A B+U \sim A B+I \sim I B+A \sim A I+B .
$$

The $2^{2} \times 5=20$ integer-valued functions just described belong to equivalence classes of size at least 5 . The remaining two integer-valued roundings of the generic translation are $T_{0}=I I+D$ and $T_{1}=I I+U$. Let $T_{2}=D D+I, T_{3}=D U+I, T_{4}=U D+I, T_{5}=U U+I$. Our discussion so far shows that any integer-valued rounding of the generic translation is equivalent to at least one of the functions $T_{i}, 0 \leq i \leq 5$. It can be checked that at any particular $(b, x) \in \mathbb{R}^{2}$ these 6 functions have at most three distinct values. Hence to verify that all six are inequivalent, we have to evaluate them at several pairs $(b, x)$. For $(b, x) \in \mathbb{R}^{2}$ and $n \in \mathbb{Z}$, let $E(b, x, n)=\left\{i: T_{i}(b, x)=n\right\}$. Then

$$
\begin{aligned}
& E(1,1.5,2)=\{0,2,4\}, \quad E(1,1.5,3)=\{1,3,5\}, \\
& E(1.5,1,2)=\{0,2,3\}, \quad E(1.5,1,3)=\{1,4,5\},
\end{aligned}
$$

and

$$
E(1.5,1.5,2)=\{2\}, \quad E(1.5,1.5,3)=\{0,1,3,4\}, \quad E(1.5,1.5,2)=\{5\} .
$$

Since any two indices occur in different sets for at least one of these three choices of $(b, x)$, it follows that no two of the functions $T_{i}, 0 \leq i \leq 5$ are equivalent. We choose them as the representatives of their equivalence classes, which must be singletons for $T_{0}$ and $T_{1}$, and 5 -sets for $T_{i}, 2 \leq i \leq 5$. Hence we have

Theorem 2. The generic translation $T(b, x)=b+x$ has 11 inequivalent roundings. They form 6 equivalence classes of integer-valued functions (2 singletons and 4 classes of size 5), and 5 equivalence classes of noninteger-valued functions (all singletons). 
TABLE 2: Roundings of $T(b, x)=b+x$

Total: 11 functions

$$
\begin{array}{cc}
\begin{array}{c}
\text { Integer-valued functions } \\
\text { Total: } 6 \text { functions }
\end{array} & \begin{array}{c}
\text { Noninteger-valued functions } \\
\text { Total: } 5 \text { functions }
\end{array} \\
2 \text { singletons: } & 5 \text { singletons: } \\
\lfloor b+x\rfloor,\lceil b+x\rceil . & b+x, \\
& b+\lfloor x\rfloor, b+\lceil x\rceil, \\
4 \text { classes of size } 5: & \lfloor b\rfloor+x,\lceil b\rceil+x . \\
\lfloor b\rfloor+\lfloor x\rfloor,\lfloor b\rfloor+\lceil x\rceil, & \\
\lceil b\rceil+\lfloor x\rfloor,\lceil b\rceil+\lceil x\rceil . &
\end{array}
$$

A representative of each of the 11 equivalence classes is given in traditional notation in Table 2. The equivalence classes of the generic translation with integer parameter, $T: \mathbb{Z} \times \mathbb{R} \rightarrow \mathbb{R}$ defined by $T(n, x)=n x+$, can readily be deduced from the above results. The restriction of the parameter to $\mathbb{Z}$ results in clusters of three formally distinct functions becoming equivalent, and we have

Corollary 2.1. The generic translation $T(n, x)=n+x$ with integer parameter $n$ has 3 inequivalent roundings. They form 2 equivalence classes of integer-valued functions (both of size 12) and one size 3 equivalence class of noninteger-valued functions. The classes have representatives $n+\lfloor x\rfloor, n+\lceil x\rceil$ and $n+x$, respectively.

As with rounded generic scale functions, we distinguish mid and final roundings of the generic translation. From Table 2 we have

Corollary 2.2. The generic translation $T(b, x)=b+x$ has 9 inequivalent mid roundings (all equivalence classes are singletons) and 6 inequivalent final roundings (2 singletons and 4 equivalence classes of size 4). Exactly 4 equivalence classes of mid roundings are integer-valued.

\section{$5 \quad$ Rounded linear functions}

Now we are ready to discuss the generic linear function $L(a, b, x)=a x * b+$. First note that it is a composition of the generic scale function $S(a, x)=a x *$ with the generic translation $T(b, x)=b x+$, in the following sense: inputting $S(a, x)=a x *$ into the second argument of $T(b, x)=b x+$ yields

$$
T(b, S(a, x))=b a x *+=a x * b+=L(a, b, x),
$$

using commutativity of additon. More generally, let us now take $S$ to be any rounded generic scale function $S=R_{0} R_{1} * R$, and $T$ to be any rounded generic translation $T=$ 
$R_{3} R^{\prime}+R_{4}$, with $R, R^{\prime}, R_{i} \in\{I, D, U\}, 0 \leq i \leq 4, i \neq 2$. Then the corresponding composition yields

$$
T(b, S(a, x))=b R_{3} a R_{0} x R_{1} * R R^{\prime}+R_{4} \sim a R_{0} x R_{1} * R_{2} b R_{3}+R_{4}=L(a, b, x)
$$

where $R R^{\prime}=R_{2} \in\{I, D, U\}$ and $L=R_{0} R_{1} * R_{2} R_{3}+R_{4}$. Recall that $R_{2}=R^{\prime}$ if $R=I$ and $R_{2}=R$ if $R \neq I$. Thus the resulting composition function $L$ is a rounded generic linear function. Conversely, given any rounded generic linear function $L$, we can express it as the composition of a suitable rounded generic scale function and rounded generic translation. Thus the results of the two previous sections allow us to study all roundings of the generic linear function.

There are $3^{5}=243$ formally distinct roundings of the generic linear function. Those which are integer-valued either have $R_{4} \in\{D, U\}$, or have $R_{4}=I$ and $R_{3} \in\{D, U\}$ together with an integer-valued rounded generic scale function $R_{0} R_{1} * R_{2}$. It follows from Theorem 1 that there are $3^{4} \times 2+22 \times 2=206$ formally distinct rounded linear functions which are integer-valued, and $243-206=37$ which are not.

We can also directly count the noninteger-valued functions. They necessarily have $R_{4}=I$, and either $R_{3}=I$, or else $R_{3} \in\{D, U\}$ and the rounded generic scale function $R_{0} R_{1} * R_{2}$ is noninteger-valued. By Theorem 1 , the number of formally distinct functions of this type is $3^{3} \times 1+5 \times 2=37$.

Now consider the equivalence classes of noninteger-valued rounded generic linear functions. There are at most 19 classes with $R_{3}=I$ because Theorem 1 shows there are 19 equivalence classes of rounded generic scale functions $R_{0} R_{1} * R_{2}$, comprising 15 singletons and 4 classes of size 3 . There are at most $5 \times 2=10$ classes with $R_{3} \in\{D, U\}$ and $R_{0} R_{1} * R_{2}$ noninteger-valued, since Theorem 1 shows that the latter belong to just 5 classes, each of which is a singleton. Hence the 37 formally distinct noninteger-valued rounded generic linear functions belong to at most $19+10=29$ equivalence classes. This number is in fact realized, because the functions take on 29 distinct values when $(a, b, x)=(10.32,0.3,20.82)$, for example.

Next consider the integer-valued rounded generic linear functions. Our earlier counting showed that the formally distinct functions of this type comprise $3^{4} \times 2=162$ final roundings and $22 \times 2=44$ mid roundings. The mid roundings have $R_{3} \in\{D, U\}$ and integer-valued $R_{0} R_{1} * R_{2}$. Let us first focus attention on them.

By Corollary 1.2, there are 14 inequivalent outer rounded generic scale functions $R_{0} R_{1}$ * $A$, where $A \in\{D, U\}$. It follows from Theorem 1 that each integer-valued rounded generic scale function is equivalent to one of them, so each equivalence class of mid rounded generic linear functions contains a member of the form $R_{0} R_{1} * A B+I$, where $B \in\{D, U\}$. Thus there are at most $14 \times 2=28$ such equivalence classes. The 14 final roundings of $a x *$ all take on different values when $(a, x)=(10.32,20.82)$, for example. Values yielded by these inputs distinguish all the functions with $B=D$ and all the functions with $B=U$, but do not fully discriminate between the two families. However, for fixed $R_{0}, R_{1}$ and $A$ the two formally distinct functions with $B=D$ and $B=U$ yield identical values if $b \in \mathbb{Z}$ but distinct values if $b \notin \mathbb{Z}$. It follows that all 28 functions are inequivalent. 
To identify all members of these 28 equivalence classes, note that

$R_{0} R_{1} * A B+I \sim R_{0} R_{1} * A B+D \sim R_{0} R_{1} * A B+U \sim R_{0} R_{1} * I B+A \sim R_{0} R_{1} * A I+B$.

Thus, the $10 \times 2=20$ cases in which $R_{0} R_{1} * A$ belongs to a singleton class (Corollary 1.2) lead to equivalence classes of rounded generic linear functions which contain at least 5 formally distinct members: of these, one is a mid rounding and 4 are final roundings of $a x * b+$. In the remaining $4 \times 2=8$ cases, $R_{0} R_{1} * A$ belongs to a class of two final rounded generic scale functions (Corollary 1.2), so to a class of 3 rounded generic scale functions (Theorem 1):

$$
R_{0} R_{1} * I \sim R_{0} R_{1} * D \sim R_{0} R_{1} * U
$$

where $R_{0} R_{1} *$ is integer-valued. The $3 \times 5=15$ operator strings resulting from the earlier equivalences include 3 strings each appearing twice, so each resultant equivalence class of rounded generic linear functions contains at least 12 formally distinct members: 3 are mid roundings and 9 are final roundings of $a x * b+$.

This accounts so far for $20 \times 1+8 \times 3=44$ formally distinct mid roundings, and $20 \times 4+8 \times 9=152$ formally distinct final roundings. Thus, only $162-152=10$ formally distinct final roundings remain. These must arise from the noninteger-valued roundings of $a x+$, which comprise 5 singletons (Theorem 1), so have the form $A I * I I+B$ or $I R * I I+B$, where $R \in\{I, D, U\}$ and $A, B \in\{D, U\}$. Suitable evaluations distinguish these among each other and from the earlier 28 classes, so we have $5 \times 2=10$ further singleton equivalence classes. This accounts for all $44+152+10=206$ formally distinct integer-valued roundings of $a x * b+$. Hence

Theorem 3. The generic linear function $L(a, b, x)=a x+b$ has 67 inequivalent roundings. They form 38 equivalence classes of integer-valued functions (10 singletons, 20 classes of size 5 and 8 of size 12), and 29 equivalence classes of noninteger-valued functions (25 singletons and 4 classes of size 3).

A representative of each of the 67 equivalence classes is given in Table 3 . The table readily yields the equivalence classes of the generic linear function with two integer parameters, $L: \mathbb{Z}^{2} \times \mathbb{R} \rightarrow \mathbb{R}$, whence

Corollary 3.1. The generic linear function $L(m, n, x)=m x+n$, with integer parameters $m, n$, has 5 inequivalent roundings. They form 4 equivalence classes of integervalued functions (2 classes of size 34 and 2 of size 84) and one size 7 equivalence class of noninteger-valued functions. Representatives for these classes are $\lfloor m x\rfloor+n,\lceil m x\rceil+n$, $m\lfloor x\rfloor+n, m\lceil x\rceil+n$ and $m x+n$, respectively.

The above results yield

Corollary 3.2. The generic linear function $L(a, b, x)=a x+b$ has 57 inequivalent mid roundings (45 singletons and 12 equivalence classes of size 3) and 38 inequivalent final 
roundings (10 singletons, 20 equivalence classes of size 4, and 8 of size 9). Exactly 28 equivalence classes of mid roundings are integer-valued.

TABLE 3: Roundings of $L(a, b, x)=a x+b$

Total: 67 functions

$$
\begin{aligned}
& \begin{array}{cc}
\text { Integer-valued functions } & \text { Noninteger-valued functions } \\
\text { Total: } 38 \text { functions } & \text { Total: } 29 \text { functions }
\end{array} \\
& 10 \text { singletons: } \\
& \lfloor a x+b\rfloor,\lceil a x+b\rceil, \\
& \lfloor a\lfloor x\rfloor+b\rfloor,\lceil a\lfloor x\rfloor+b\rceil, \quad 25 \text { singletons: } \\
& \lfloor a\lceil x\rceil+b\rfloor,\lceil a\lceil x\rceil+b\rceil, \quad a x+b, \\
& \lfloor\lfloor a\rfloor x+b\rfloor,\lceil\lfloor a\rfloor x+b\rceil, \quad a\lfloor x\rfloor+b, a\lceil x\rceil+b, \\
& \lfloor\lceil a\rceil x+b\rfloor,\lceil\lceil a\rceil x+b\rceil . \quad\lfloor a\rfloor x+b,\lceil a\rceil x+b, \\
& \lfloor a x\rfloor+\lfloor b\rfloor,\lfloor a x\rfloor+\lceil b\rceil, \quad\lfloor a\lceil x\rceil\rfloor+b,\lceil a\lceil x\rceil\rceil+b, \\
& \lceil a x\rceil+\lfloor b\rfloor,\lceil a x\rceil+\lceil b\rceil, \quad\lfloor\lceil a\rceil x\rfloor+b,\lceil\lceil a\rceil x\rceil+b, \\
& \lfloor a\lfloor x\rfloor\rfloor+\lfloor b\rfloor,\lfloor a\lfloor x\rfloor\rfloor+\lceil b\rceil, \quad\lfloor a x\rfloor+b,\lceil a x\rceil+b, \\
& \lfloor a\lceil x\rceil\rfloor+\lfloor b\rfloor,\lfloor a\lceil x\rceil\rfloor+\lceil b\rceil, \quad a x+\lfloor b\rfloor, a x+\lceil b\rceil, \\
& \lceil a\lfloor x\rfloor\rceil+\lfloor b\rfloor,\lceil a\lfloor x\rfloor\rceil+\lceil b\rceil, \quad a\lfloor x\rfloor+\lfloor b\rfloor, a\lfloor x\rfloor+\lceil b\rceil, \\
& \lceil a\lceil x\rceil\rceil+\lfloor b\rfloor,\lceil a\lceil x\rceil\rceil+\lceil b\rceil, \quad a\lceil x\rceil+\lfloor b\rfloor, a\lceil x\rceil+\lceil b\rceil, \\
& \lfloor\lfloor a\rfloor x\rfloor+\lfloor b\rfloor,\lfloor\lfloor a\rfloor x\rfloor+\lceil b\rceil, \quad\lfloor a\rfloor x+\lfloor b\rfloor,\lfloor a\rfloor x+\lceil b\rceil, \\
& \lfloor\lceil a\rceil x\rfloor+\lfloor b\rfloor,\lfloor\lceil a\rceil x\rfloor+\lceil b\rceil, \quad\lceil a\rceil x+\lfloor b\rfloor,\lceil a\rceil x+\lceil b\rceil . \\
& \lceil\lfloor a\rfloor x\rceil+\lfloor b\rfloor,\lceil\lfloor a\rfloor x\rceil+\lceil b\rceil, \\
& \lceil\lceil a\rceil x\rceil+\lfloor b\rfloor,\lceil\lceil a\rceil x\rceil+\lceil b\rceil . \quad 4 \text { classes of size } 3 \text { : } \\
& 8 \text { classes of size 12: } \quad\lceil a\rceil\lfloor x\rfloor+b,\lceil a\rceil\lceil x\rceil+b . \\
& \lfloor a\rfloor\lfloor x\rfloor+\lfloor b\rfloor,\lfloor a\rfloor\lfloor x\rfloor+\lceil b\rceil, \\
& \lfloor a\rfloor\lceil x\rceil+\lfloor b\rfloor,\lfloor a\rfloor\lceil x\rceil+\lceil b\rceil, \\
& \lceil a\rceil\lfloor x\rfloor+\lfloor b\rfloor,\lceil a\rceil\lfloor x\rfloor+\lceil b\rceil \text {, } \\
& \lceil a\rceil\lceil x\rceil+\lfloor b\rfloor,\lceil a\rceil\lceil x\rceil+\lceil b\rceil \text {. } \\
& \begin{array}{l}
\lfloor a\rfloor\lfloor x\rfloor+b,\lfloor a\rfloor\lceil x\rceil+b, \\
\lceil a\rceil\lfloor x\rfloor+b,\lceil a\rceil\lceil x\rceil+b .
\end{array}
\end{aligned}
$$

\section{Some remarks and open questions}

Various related questions for rounded generic linear functions remain open. For example, one could ask for the maximum number of distinct values assumed by the integer-valued roundings of the generic linear function within 38 consecutive integers, or the length of a smallest interval in which the integer-valued roundings take on the maximum number of distinct values, or the length of a smallest interval in which all roundings take on the maximum number of distinct values.

Note that for any fixed $(a, b, x) \in \mathbb{R}^{3}$, the smallest interval $A(a, b, x)$ which contains all values assumed by the 67 inequivalent roundings of the generic linear function is a 
closed interval with integer endpoints. This follows from the observation that if $L_{0}$ and $L_{1}$ are noninteger-valued rounded generic linear functions which achieve the minimum and maximum values at $(a, b, x)$ among the 29 inequivalent noninteger-valued roundings, then $\left\lfloor L_{0}(a, b, x)\right\rfloor$ and $\left\lceil L_{1}(a, b, x)\right\rceil$ are values of integer-valued roundings, and the closed interval $B(a, b, x)$ with these integers as endpoints contains all the noninteger values assumed by the roundings at $(a, b, x)$. In fact, it is easy to check that $a D x D * b D+$ and $a U x U * b U+$ are the endpoints of $A(a, b, x)$ when $(a, x) \in\left(\mathbb{R}^{+}\right)^{2}$, and to give similar descriptions of the endpoints when $(a, x)$ is in each of the other three quadrants of $\mathbb{R}^{2}$. When is $A(a, b, x)$ strictly larger than $B(a, b, x)$ ?

We have discussed the generic linear function $L: \mathbb{R}^{3} \rightarrow \mathbb{R}$ defined by $L(a, b, x)=$ $a x * b+$ in reverse Polish notation, and decomposed it into the generic scale function $S(a, x)=a x *$ followed by the generic translation $T(b, x)=b x+$. If we compose these two functions in the reverse order we get

$$
S(a, T(c, x))=a c x+*=a x * a c *+=L(a, a c *, x),
$$

using commutative and distributive properties. The composition in this order almost gives the generic linear function, but not quite: there is no choice of $(a, c, x) \in \mathbb{R}^{3}$ to correspond to $L(0, b, x)$ with $b \neq 0$. But one can still study the near-generic linear function $M: \mathbb{R}^{3} \rightarrow \mathbb{R}$ defined by $M(a, c, x)=a c x+*$. Note that $M(a, c, x)=c x+a *$, a formulation which more closely parallels our specification for $L(a, b, x)$. Then any rounded near-generic linear function is a function $M: \mathbb{R}^{3} \rightarrow \mathbb{R}$ defined by

$$
M(a, c, x)=c R_{0} x R_{1}+R_{2} a R_{3} * R_{4}
$$

where $R_{i} \in\{I, D, U\}, 0 \leq i \leq 4$. For brevity, $M=R_{0} R_{1}+R_{2} R_{3} * R_{4}$. Once again, there are $3^{5}=243$ formally distinct functions of this type. It might be supposed that these

rounded functions would belong to equivalence classes of the same number and spectrum of sizes as those described in Theorem 3 for roundings of the generic linear function. However, it may actually turn out that the near-generic linear function $M(a, c, x)=(c+x) a$ has 75 inequivalent roundings, distributed across 54 equivalence classes of integer-valued functions (34 singletons, 4 classes of size 3, 8 of size 5 and 8 of size 15) and 21 equivalence classes of noninteger-valued functions (17 singletons and 4 classes of size 5 ). In the interests of brevity any proof of this claim is omitted, so the reader may treat it as a conjecture rather than a result.

The author gratefully acknowledges hospitality of the Mathematics Department, University of Newcastle, Australia where this paper was written during his sabbatical leave in early 2000.

\section{References}

[1] S. Beatty, Problem 3177, Amer. Math. Monthly 34 (1927), 159-160.

[2] L.E. Dickson, History of the Theory of Numbers, vol. 1 (1918), Chelsea reprint (1952). 
[3] R.B. Eggleton, A.S. Fraenkel, R.J. Simpson, Beatty sequences and Langford sequences, Discrete Math. 111 (1993), 165-178.

[4] A.S. Fraenkel, Iterated floor function, algebraic numbers, discrete chaos, Beatty subsequences, semigroups, Trans. Amer. Math. Soc. 341 (1994), 639-664.

[5] R.L. Graham, D.E. Knuth, O. Patashnik, Concrete Mathematics, Addison-Wesley (1988).

[6] I.J. Håland, Uniform distribution of generalized polynomials, J. Number Theory 45 (1993), 327-366.

[7] G.H. Hardy and E.M. Wright, An Introduction to the Theory of Numbers, ed. 4, Clarendon Press (1960).

[8] K.E. Iverson, A Programming Language, Wiley (1962).

[9] E. Kitcher, Problem 1300, Math. Mag. 61 (1988), 196. Solution by J. Propp, Math. Mag. 62 (1989), 203.

[10] D.E. Knuth, The Art of Computer Programming, v.1, Fundamental Algorithms, ed. 3, Addison-Wesley (1997).

[11] A.M. Legendre, Théorie des nombres, ed. 2 (1808). 